\title{
Caracterização mineralógica dos Lutitos Diatomáceos da Península de Santa Elena, Equador
}

\author{
(Mineralogical characterization of the Diatoms Lutites \\ of the Santa Elena Peninsula, Ecuador)
}

\section{Miguel Genaro Peralta Sánchez \\ Mestrando, DEGEO/EM/UFOP. \\ Ouro Preto/Brasil \\ Pesquisador Componente 6 . VLIR/ESPOL. FICT/ESPOL. \\ Guaiaquil-Equador \\ E-mail:miguelp@degeo.ufop.br}

\section{Newton Souza Gomes \\ Professor do DEGEO/EM/UFOP \\ Ouro Preto/Brasil \\ E-mail:newton@degeo.ufop.br}

\section{Mauricio Antônio Carneiro \\ Professor do DEGEO/EM/UFOP \\ Ouro Preto/Brasil \\ E-mail:mauricio@degeo.ufop.br}

\section{Marcos Andrade Gonçalves \\ Professor do DEQUI/ICEB/UFOP \\ Ouro Preto/Brasil \\ E-mail: marcos.andrade@iceb.ufop.br}

\author{
Maria Paula Delicio \\ Professora do DEGEO/EM/UFOP \\ Ouro Preto/Brasil \\ E-mail:mpaula@degeo.ufop.br
}

\section{Resumo}

Esse trabalho apresenta as principais características mineralógicas, físicas e geoquímicas dos lutitos diatomáceos do Membro Villingota da Formação Tosagua, localizada na bacia Progreso no sudoeste do litoral do Equador, visando à sua utilização como matéria-prima na elaboração de compostos cerâmicos mediante ativação alcalina. Os pelitos estudados do Membro Villingota apresentam uma complexa mistura que se caracteriza pela composição química, na qual predominam sílica, carbonato e alumina, e por sua variada fauna de microfósseis, composta de diatomáceas, radiolários, espículas e foraminíferos. A ativação alcalina dessa mistura natural possibilitou a fabricação de geopolímeros, considerados materiais inorgânicos que podem substituir as argamassas e produtos minerais.

Palavras-chave: Lutitos diatomáceos, microfósseis, Península Santa Elena, Membro Villingota.

\section{Abstract}

This work describes the main mineralogical, physical and geochemical characteristics of the diatom lutites of the Villingota Member of the Tosagua Formation, located in the Progreso Basin in the Southwest of the Equatorian coast, with the view of using them as raw materials in the ceramic industry by means of alkaline activation. The studied pelites of the Villingota Member are composed of a complex mixture characterized by their chemical composition with predominance of silica, carbonate, and alumina, as well as by their varied microfossil fauna that includes diatoms, radiolarians, spicules and foraminifers. The alkaline activation of this natural mixture turned possible the manufacture of geopolymers, which are inorganic materials that can substitute binders and mineral products.

Keywords: Diatoms lutite, microfossil Santa Elena Peninsula, Villingota Member. 


\section{Introdução}

A Península de Santa Elena localiza-se no sudoeste do Equador e constitui uma importante unidade fisiográfica da Ámerica do Sul. Estratigraficamente compreende uma seqüência de rochas ígneas cretácicas na base, sobrepostas por rochas sedimentares terciárias e com coberturas quaternárias. Essa unidade foi alvo de algumas pesquisas científicas e tecnológicas anteriores, sendo as mais importantes: a exploração de hidrocarbonetos (Higley, 2004; Anglo-Ecuadorian Oilfields, 1970), matéria-prima para cimento portland (Holderbank, 1974), rochas e minerais industriais (PeraltaSánchez, 2008; Morales-Carrera, 2008; Morales-Carrera, 2003; Ramos, 1996; Carballo, 1994; Arrata et al., 1990; Brito \& Markwich, 1990) e micropaleontológicas (Ordoñez et al., 2006; Ordoñez et al., 1986). Os lutitos diatomáceos estudados correspondem aos pelitos tipo do Membro Villingota da Formação Tosagua localizada no extremo oeste da bacia Progreso.

Os trabalhos realizados no principal depósito de lutitos diatomáceos, perto do povoado de Sucre por Ramos (1996) e Arrata et al. (1990), orientaram a utilização desses lutitos na fabricação de tijolos leves. Essa atividade, entretanto, não prosperou na região.

Os lutitos caracterizados nesse trabalho com o objetivo de conhecer suas principais características mineralógicas, químicas, físicas e texturais foram obtidos de três ocorrências localizadas no Membro Villingota. Os teores de sílica, alumina e cal, relacionados à presença de argila, sílica e calcita biogênica, feldspatos, quartzo e zeólitas, permitiram considerar essas misturas naturais como possível matéria-prima na obtenção de estruturas geopoliméricas pelo método proposto por Xu e Deventer (2000), que consiste no aquecimento do material a temperaturas entre $500^{\circ} \mathrm{C}$ e $900^{\circ} \mathrm{C}$ e logo a sua ativação com soluções alcalinas (Davidovits, 1982).

\section{Contexto Geológico}

A Península de Santa Elena como toda a Costa Equatoriana é constituída por terrenos sedimentares alóctones, acrescentados durante o Paleoceno sobre um embasamento ígneo-basáltico e basáltico-andesítico, que pertenceu a uma antiga crosta oceânica (Goosens et al., 1973; Juteau et al., 1977; Feininger \& Bristow, 1980; Mégard et al., 1987), formada no Cretáceo Inferior (Deniaud et al., 1998). Esses terrenos emergiram no Eoceno Tardio (Benitez, 1995; Jaillard et al., 1995). A geodinâmica em etapas do arco-frontal (fore-arc), provocada pelo sistema de subducção da placa de Nazca abaixo da placa Sul-Americana (Lonsdale, 1978; Dorfman, 1976), foi que permitiu a acrescão desses terrenos alóctones (Benitez, 1995).

$\mathrm{Na}$ zona central da Península de Santa Elena, localiza-se a bacia $\mathrm{Ne}$ ogena Progreso, definida como uma depressão sinclinal, gerada por uma subsidência rápida no Oligoceno Tardio e com posterior preenchimento de sedimentos Oligo-Miocênicos (Ordoñez et al., 2006). A bacia possui uma área aproximada de $3200 \mathrm{~km}^{2}$ e representa uma zona deprimida do arco-frontal, limitado ao norte pela Cordilheira de Chongón-Colonche, ao sul-oeste pelo soerguimento de Santa Elena e ao este com o Golfo de Guayaquil (Figura 1A).

Na bacia Progreso são encontradas rochas sedimentares dos Grupos Azúcar e Ancón, assim como das Formações Tosagua, Subibaja, Progreso, Puná e Tablazo.

A Formação Tosagua, de idades compreendidas entre o Oligoceno Superior e Mioceno Médio, é formada pelos membros Zapotal, Dos Bocas e Villingota (Figura 1B).

O Membro Villingota consiste de lutitos laminados diatomáceos, macios e leves, de cor branca devido à meteorização. A espessura desse membro varia entre 250 e 650 metros (Baldock, 1982).

A microfauna encontrada no Membro Villingota é variada e consiste, principalmente, de foraminíferos, restos de peixes, espículas silicosas, carapaças de diatomáceas e radiolários. Em face disto, infere-se que a deposição desse membro ocorreu em um ambiente marinho de plataforma externa a talude continental (Ordoñez et al., 2006).

\section{Materiais e métodos}

A área de estudo inclui três ocorrências de lutitos diatomáceos dentro da Formação Villingota, denominadas como A01, A02 e A03, entre os povoados de Sucre, Olmedo e San Antonio, onde foram coletadas cinco amostras denominadas NM_23A, NM_13, NM_21A, NM_29 e NM_43B (Tabela 1). As amostras coletadas apresentaram características mineralógicas e fossilíferas similares.

O teor de umidade das amostras foi determinado com a utilização de uma estufa artesanal e de uma balança da SARTORIUS modelo 1602 MP81. Na metodologia utilizada, foram pesados $100 \mathrm{~g}$ de lutitos diatomáceos, posteriormente secados a $70^{\circ} \mathrm{C}$, durante 24 horas, e novamente pesados para se obter o percentual de umidade.

As análises granulométricas foram realizadas seguindo o método da pipeta para solos normais proposto pela EMBRAPA (1997), o qual se baseia na Lei de Stokes. As análises de difração de raios $\mathrm{X}$ foram feitas no laboratório raios-X/DEGEO/UFOP, utilizando-se um difratômetro de marca RIGAKU, modelo GEIGERFLEX D/MAX, com goniômetro horizontal, tubo de $\mathrm{Cu}$, velocidade de $1,2^{\circ}$ por segundo, com varredura de 2 a $70^{\circ}$, para a fração total (amostra natural), e velocidade de $0,6^{\circ}$ por segundo, com varredura de 2 a $35^{\circ}$, para a fração argila $(\Phi<2 \mu \mathrm{m})$.

As análises químicas das amostras de lutitos diatomáceos foram realizadas no laboratório LFR-X/DEGEO/UFOP em um aparelho de fluorescência de raios $\mathrm{X}$ de marca PHILLIPS, modelo MAGI X. A densidade de cada amostra foi quantificada em um Multipicnômetro da QUANTACHROME. As análises de superfície específica pelo 
método de B.E.T. (Brunauer, Emmett e Teller, 1938) e de porosidade foram feitas no Laboratório de Metalurgia do Departamento de Metalurgia (DEMET) da UFOP, utilizando-se um equipamento High Speed Gas Sorption de marca QUANTACHROME, modelo NOVA 1000 , com adsorção de $\mathrm{N}_{2}$, desgasificação a $60^{\circ} \mathrm{C}$, durante 5 horas, e adsorção de $\mathrm{N}_{2}$ a $-196^{\circ} \mathrm{C}$. As curvas termodiferenciais (ATD) e termogravimétricas (ATG) foram obtidas com um equipamento de marca TA INSTRUMENTS, modelo 2960 STD V2.2B, usando um portaamostra de platina e de alumina (padrão), com taxa de aquecimento de $20^{\circ} \mathrm{C}$ por minuto, em atmosfera de oxigênio até uma temperatura máxima de $900^{\circ} \mathrm{C}$. $\mathrm{Na}$ análise de microscopia ótica realizada no laboratório de Microscopia Ótica/ DEGEO/UFOP, observaram-se, principalmente, os microfósseis presentes nas amostras. Utilizou-se o microscópio ótico trinocular de marca Zeiss, modelo Axiophot, equipado com câmara digital. Nas análises de microscopia eletrônica de varredura (MEV) /DEGEO/UFOP, obtiveram-se imagens morfológicas tridimensionais de detritos e dos microfósseis através de um microscópio eletrônico de marca JEOL JSM-5510, de 0.5 a $30 \mathrm{kV}$, com resolução de 3,5 a $48 \mathrm{~nm}$, com recobrimento de grafita.

As amostras foram aquecidas a temperaturas entre $550^{\circ} \mathrm{C}$ e $800^{\circ} \mathrm{C}$, tendo sido misturadas e homogeneizadas. Essas misturas foram umedecidas com soluções alcalinas de $\mathrm{NaOH}$ a $15 \mathrm{~mol} / 1$, homogeneizadas, confinadas e colocadas em estufa com temperaturas menores que $50^{\circ} \mathrm{C}$, para acelerar a reação e não danificar os copos-de-prova. Decorridas três horas e após se verificar a geopolimerização, as amostras foram submetidas à temperatura ambiente até dez dias, para se obter o endurecimento final do material. As quantidades de solução alcalina misturada à amostra foram calculadas segundo a relação molar $\mathrm{Na}_{2} \mathrm{O}$ / $\mathrm{Al}_{2} \mathrm{O}_{3}$ total da mistura (matéria-prima e reagente) atinja a faixa entre 1,00 a 1,14 (Davidovits, 1982). Também foi considerada a relação molar $\mathrm{SiO}_{2} / \mathrm{Al}_{2} \mathrm{O}_{3}$ da mistura.

Os corpos-de-prova foram submetidos à compressão simples no
Laboratório de Ensaios Mecânicos do Departamento de Metalurgia (DEMETUFOP), utilizando-se um equipo hidráulico MTS-850 com aquisição de dados digital.

\section{Resultados e discussão}

A umidade média quantificada, nas amostras coletadas, nas três ocorrências, é de $8,81 \%$. A densidade aparente média das medições in situ foi de $1,92 \mathrm{~g} / \mathrm{cm}^{3}$.

As análises granulométricas realizadas visaram a quantificar os percentuais das três principais frações contidas: areia $(62<\phi<2000 \mu \mathrm{m})$, silte $(2<\phi<$ $62 \mu \mathrm{m})$ e argila $(\phi<2 \mu \mathrm{m})$. Em todas as amostras predomina a fração silte, com valores entre 58 e $92 \%$ (Figura 2). Esses altos valores incluem partículas aglomeradas da fração argila, pois é comum a aglomeração de argilominerais, devido à cimentação por carbonatos, tal como pode ser observado por microscopia eletrônica de varredura.

As análises químicas das cinco amostras de lutitos diatomáceos naturais e de sua mistura permitiram definir os teores (\% em peso) dos óxidos de maior proporção $\mathrm{SiO}_{2}, \mathrm{Al}_{2} \mathrm{O}_{3}, \mathrm{CaO}, \mathrm{Fe}_{2} \mathrm{O}_{3} \mathrm{e}$ $\mathrm{Na}_{2} \mathrm{O}$ e dos menores $\mathrm{TiO}_{2}, \mathrm{MgO}, \mathrm{K}_{2} \mathrm{O}$ e $\mathrm{P}_{2} \mathrm{O}_{5}$. A Tabela 1 indica a composição química das cinco amostras. A variação dos percentuais de $\mathrm{SiO}_{2}$ entre aproximadamente 44,16 e $67,09 \%$ está relacionada com a presença de quartzo

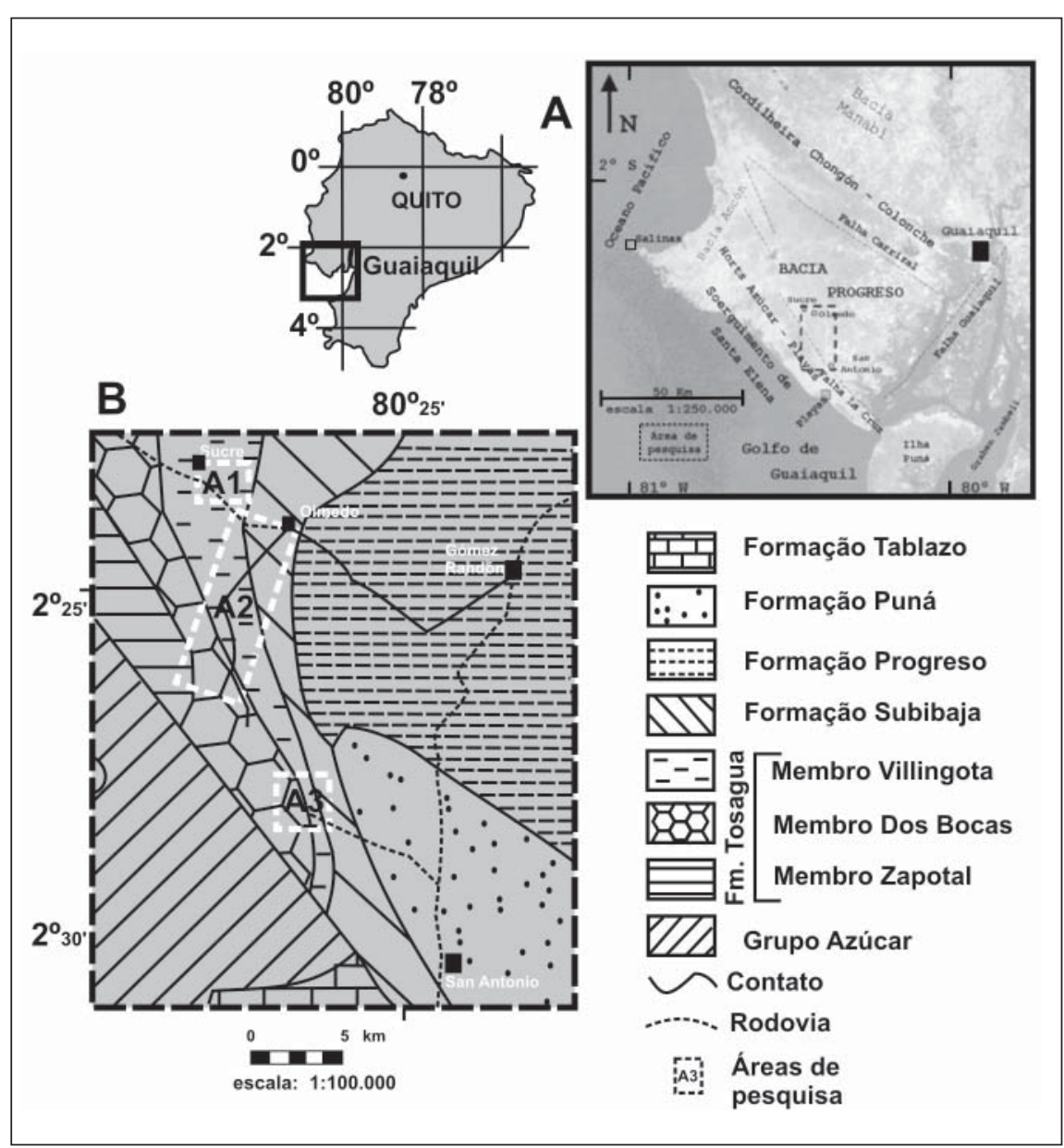

Figura 1 - A: Localização da bacia Progreso - área de pesquisa na Península de Santa Elena sudoeste do Equador (modificado ESDI, 2004; Deniaud et al., 1999). B: Localização das áreas de pesquisa A1, A2 e A3, no Membro Villingota da Formação Tosagua na Península de Santa Elena-Equador (modificado ESDI, 2004; IGM, 1984; IGM, 1968). 
Tabela 1 - Composição química (\%) em peso via Fluorescência de Raios X das cinco amostras de lutitos diatomáceos e da média obtida da mistura dessas cinco amostras.

\begin{tabular}{c|c|c|c|c|c|c|c|c|c|c|c|c}
\hline \multicolumn{2}{c|}{ Amostra } & $\mathrm{SiO}_{2}$ & $\mathrm{Al}_{2} \mathrm{O}_{3}$ & $\mathrm{CaO}$ & $\mathrm{Fe}_{2} \mathrm{O}_{3}$ & $\mathrm{Na}_{2} \mathrm{O}$ & $\mathrm{TiO}_{2}$ & $\mathrm{MgO}$ & $\mathrm{K}_{2} \mathrm{O}$ & $\mathrm{P}_{2} \mathrm{O}_{5}$ & P.F. & Total \\
\hline \multirow{2}{*}{ Área 1 } & NM_23A & 67,09 & 9,36 & 4,85 & 3,55 & 3,46 & 0,95 & 0,95 & 0,51 & 0,20 & 9,07 & 99,99 \\
\cline { 2 - 13 } & NM_13 & 56,10 & 13,26 & 8,20 & 4,63 & 1,65 & 1,31 & 1,31 & 0,62 & 0,39 & 12,53 & 100,00 \\
\hline \multirow{2}{*}{ Área 2 } & NM_21A & 49,92 & 14,91 & 9,35 & 5,39 & 2,60 & 1,55 & 1,54 & 0,55 & 0,19 & 13,99 & 99,99 \\
\cline { 2 - 12 } & NM_29 & 61,16 & 12,32 & 5,73 & 5,66 & 1,59 & 0,52 & 1,73 & 0,61 & 0,05 & 10,62 & 99,99 \\
\hline Área 3 & NM_43B & 44,16 & 11,97 & 15,58 & 3,93 & 3,63 & 1,56 & 1,57 & 0,52 & 0,45 & 16,62 & 99,99 \\
\hline \multicolumn{2}{l}{ Média } & 58,30 & 12,01 & 8,00 & 3,90 & 1,90 & 1,02 & 1,40 & 0,55 & 0,28 & 12,64 & 100,00 \\
\hline
\end{tabular}

e de feldspatos, além da sílica amorfa dos microfósseis silicosos tais como as diatomáceas. Os percentuais de $\mathrm{Al}_{2} \mathrm{O}_{3}$ variam entre 9,36 e $14,91 \%$ e podem ser relacionados com a presença de argilominerais. Já os teores de $\mathrm{CaO}$, que variam entre 4,85 e 15,58\%, correspondem à presença de carbonatos e microfósseis como foraminíferos.

As análises por difração de raios $\mathrm{X}$ identificaram as espécies minerais presentes nas cinco amostras de lutitos diatomáceos. Os difratogramas mostraram uma mineralogia similar em todas as amostras, que é formada por minerais argilosos e, principalmente, de nãoargilosos. Essa variedade mineralógica dificultou a exata identificação dos minerais presentes, sendo que alguns picos podem indicar a presença de mais de um mineral. As maiores proporções de minerais encontradas nas amostras, em ordem decrescente são: calcita, quartzo, argilomineral do grupo da esmectita e feldspatos. Os argilominerais do grupo da caulinita, mica e zeólitas aparecem como traços. Na Figura 3, têm-se os difratogramas das cinco amostras.

Devido à freqüente presença de minerais argilosos nos depósitos, foi realizada difração de raios $\mathrm{X}$ da fração argila. Nos difratogramas obtidos na fração argila das amostras de lutitos diatomáceos naturais, após saturações com $\mathrm{MgCl}_{2}$ e $\mathrm{KCl}$, segundo a metodologia de Walker (1958), e, após saturação com LiCl, segundo a metodologia proposta por Greene-Kelly (1953), foi

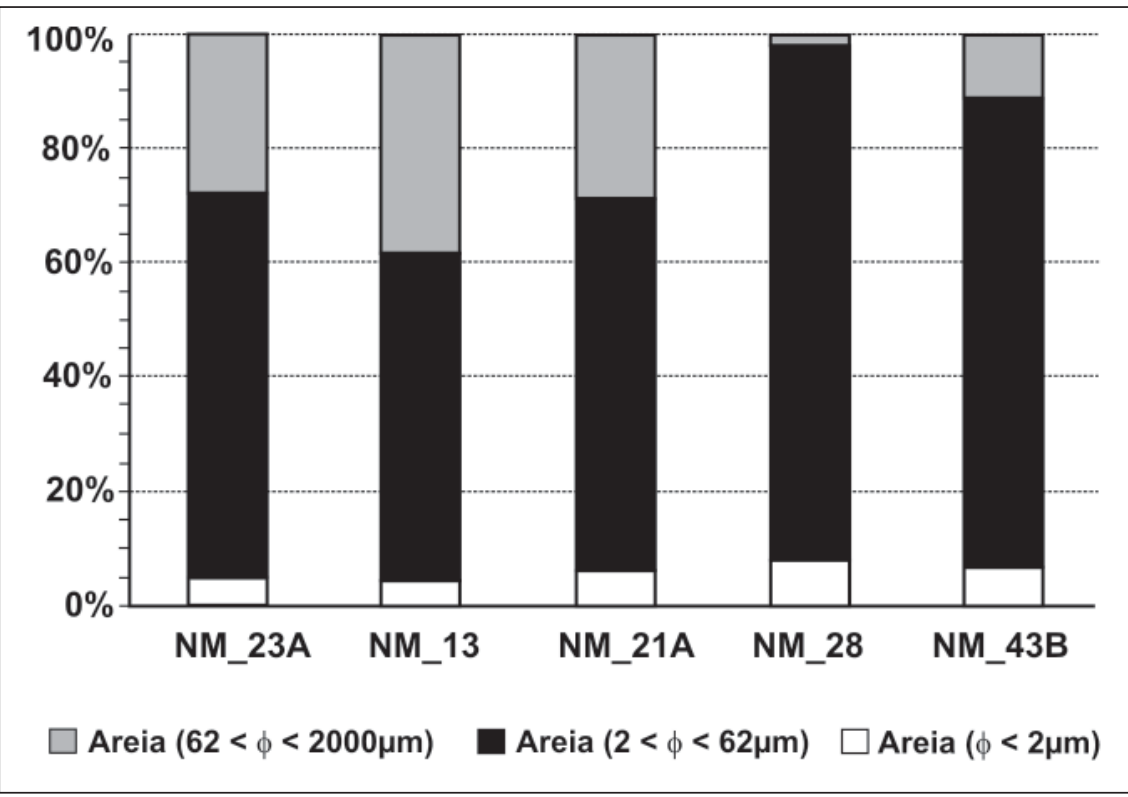

Figura 2 - Distribuição granulométrica das cinco amostras de lutitos diatomáceos.

possível identificar o grupo e a espécie do principal argilomineral presente em todas as amostras. A Figura 4A indica os difratogramas da fração argila da amostra NM_13 natural nas seguintes condições: secada ao ambiente, saturada com etileno glicol e calcinada. Quando a amostra foi secada ao ambiente, o valor do espaço interplanar basal (d001) da estrutura cristalina do argilomineral é de $12,8 \AA$, após saturação com etileno glicol a estrutura cristalina expande e d aumenta para $14,6 \AA \mathrm{e}$, depois de calcinada a $500^{\circ} \mathrm{C}$, a estrutura cristalina colapsa a $9,5 \AA$. Essas mudanças na estrutura cristalina indicam que o argilomineral é expansível. Após saturar com íons de magnésio e com etileno glicol (Figura 4B), o valor de d situa-se próximo a $18,5 \AA$. No caso da saturação com íons de potássio e com etileno glicol, o valor de d situa-se próximo a $15,7 \AA$. Esses valores de d são próximos aos sugeridos por Walker (1958), para os argilominerais do grupo das esmectitas. Na Figura 4C, pode-se observar que o valor $\mathrm{d}$ inicial de $15,2 \AA$ na amostra natural diminuiu após saturação com $\mathrm{LiCl}$, aquecimento a $300^{\circ} \mathrm{C}$ e saturação com etileno glicol até $10,3 \AA$, resultando em um valor próximo do sugerido por Greene-Kelly (1953), concluindo-se, assim, que o principal argilomineral contido nos lutitos diatomáceos é uma montmorilonita. 
As análises termodiferenciais (ATD), representadas na Figura 5, mostram o comportamento térmico das cinco amostras naturais de lutitos diatomáceos. Pode-se reconhecer a presença de minerais argilosos e outros não argilosos que possuem a propriedade de adsorver água. Assim, os dois primeiros picos endotérmicos que aparecem a temperaturas entre $53^{\circ} \mathrm{C}$ e $145^{\circ} \mathrm{C}$ são relativos à perda de água adsorvida nas amostras e são, tipicamente, encontrados no argilomineral montmorilonita. Os dois picos endotérmicos seguintes, entre $463^{\circ} \mathrm{C}$ e $705^{\circ} \mathrm{C}$, referem-se à perda da água das hidroxilas da estrutura cristalina da montmorilonita. $\mathrm{O}$ pico endotérmico que aparece entre $463^{\circ} \mathrm{C} \mathrm{e} 477^{\circ} \mathrm{C}$ representa a presença também de caulinita, enquanto que o pico endotérmico entre $675^{\circ} \mathrm{C}$ e $705^{\circ} \mathrm{C}$ também indica a presença de carbonatos (Mackenzie, 1957).

$\mathrm{Na}$ Tabela 2, são apresentados os valores de densidade, superfície específica e porosidade das cinco amostras de lutitos diatomáceos. Os valores de densidade aparente variam entre 1,84 e $2,06 \mathrm{~g} / \mathrm{cm}^{3}$ e os da densidade específica

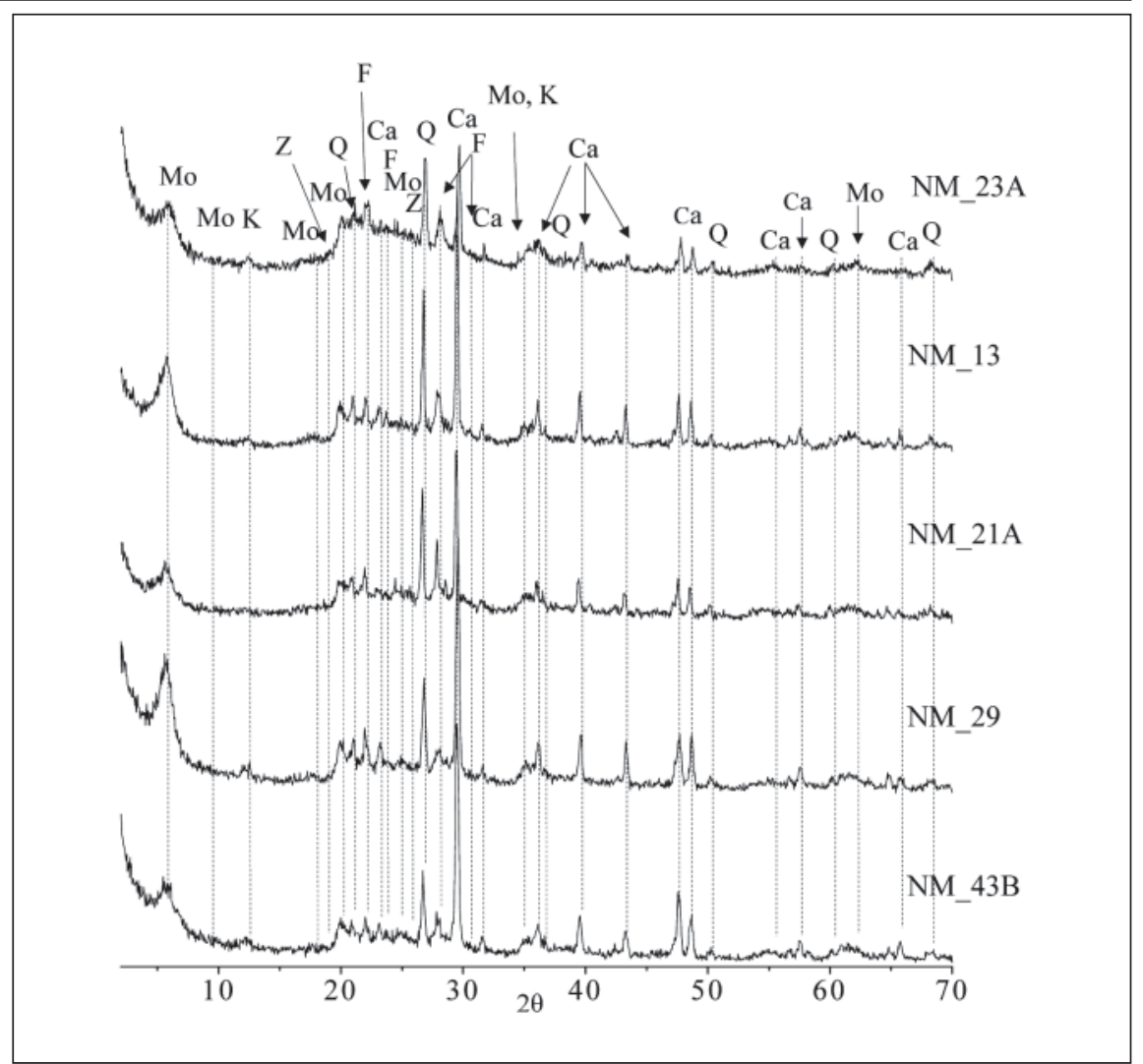

Figura 3 - Difratogramas de cinco amostras de lutitos diatomáceos, indicando a mineralogia contida (Mo: montmorilonita, K: caulinita, Q: quartzo, F: feldspato, Ca: calcita, Z: zeólita).
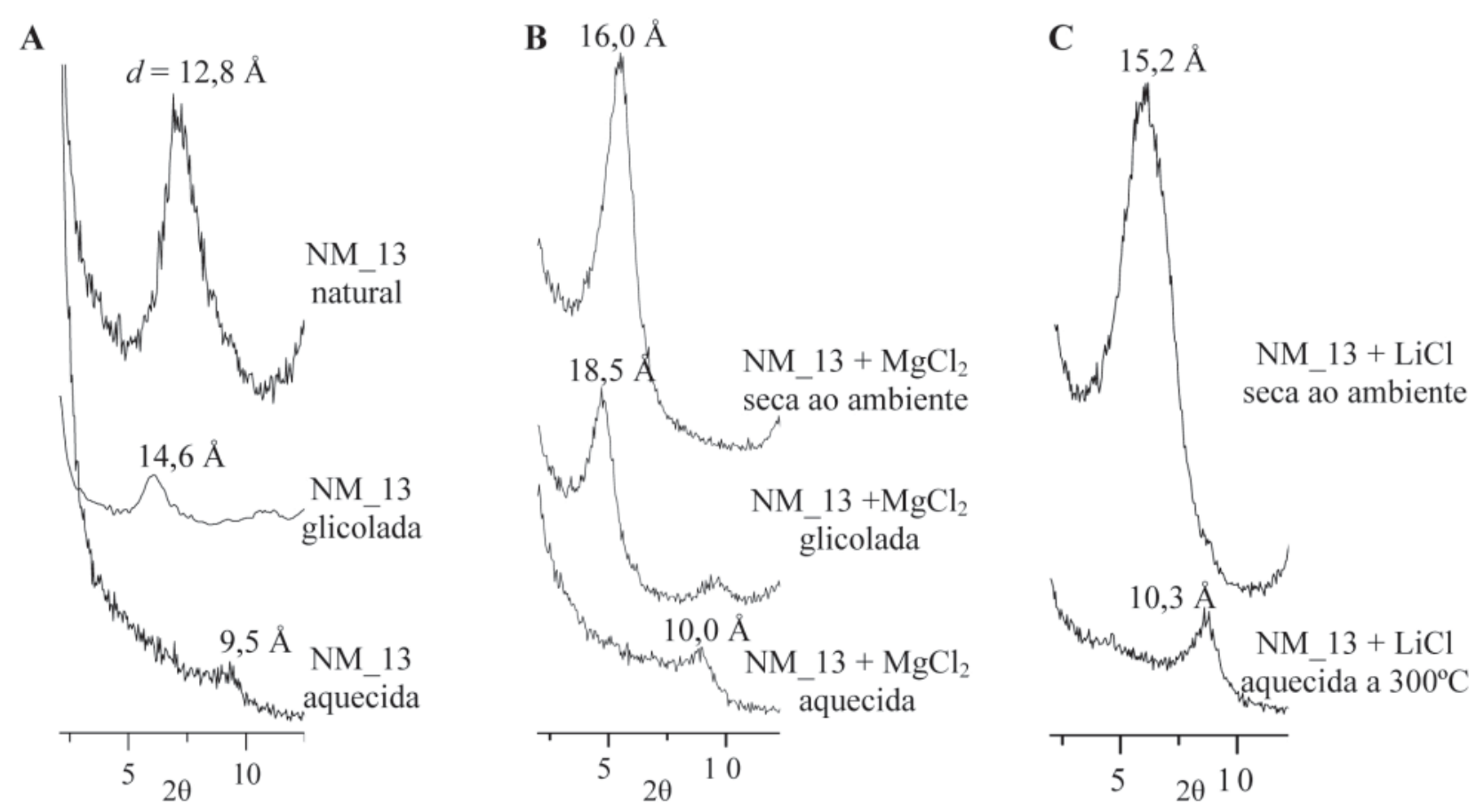

Figura 4 - Difratogramas da fração argila da amostra NM_13 A: natural, glicolada e aquecida; B: saturada com MgCl ; C: com LiCl. 
variam entre 2,07 e $2,42 \mathrm{~g} / \mathrm{cm}^{3}$. Esses valores representam a contribuição dos diferentes minerais identificados por difratogramas de raios X. Em geral, a menor densidade estaria relacionada à maior proporção de sílica amorfa e montmorilonita.

Os valores de superfície específica variam entre 8,11 e $20,24 \mathrm{~m}^{2} / \mathrm{g}$. Esses valores poderiam estar relacionados, respectivamente, ao menor e ao maior conteúdo do argilomineral montmorilonita nas amostras, porém a influência dos demais minerais presentes resulta em valores mais baixos que os dos esperados para a montmorilonita pura.

O volume total de poros das amostras é variável, desde o valor mínimo de $0,001 \mathrm{~cm}^{3} / \mathrm{g}$ para a amostra NM_29, da ocorrência A03 até $0,076 \mathrm{~cm}^{3} / \mathrm{g}$ para a amostra NM_13 da ocorrência A01.

As análises de microscopia ótica e eletrônica de varredura permitiram observar a morfologia e a estrutura dos microfósseis presentes nas amostras estudadas (Figura 6). Na área A01, foram observadas diatomáceas, tanto em suas formas completas, como também em fragmentos. Os radiolários foram encontrados, predominantemente, em fragmentos. Nas áreas A02 e A03, os radiolários são, relativamente, mais abundantes do que as diatomáceas e identificaram-se, também, abundantes fragmentos silicosos, que poderiam ser de uma mistura de vários tipos de microfósseis. Estima-se que a massa total dos microfósseis silicosos varia entre $10 \mathrm{e}$ $20 \%$ e a das diatomáceas, entre 3 e 10\%. Entre os microfósseis de diatomáceas foram identificados os tipos marinhos Pennales e Centrales (Vilela, 2004). Entre os microfósseis de radiolários, foram identificados os pertencentes às ordens Nassellaria e Spumellaria (Eilert et al., 2004). Também foram observados microfósseis calcíticos de foraminíferos, principalmente do tipo enrolado involuto e reto bisseriado (Cushman, 1976).

O material obtido da mistura das cinco amostras foi aquecido à tempera-

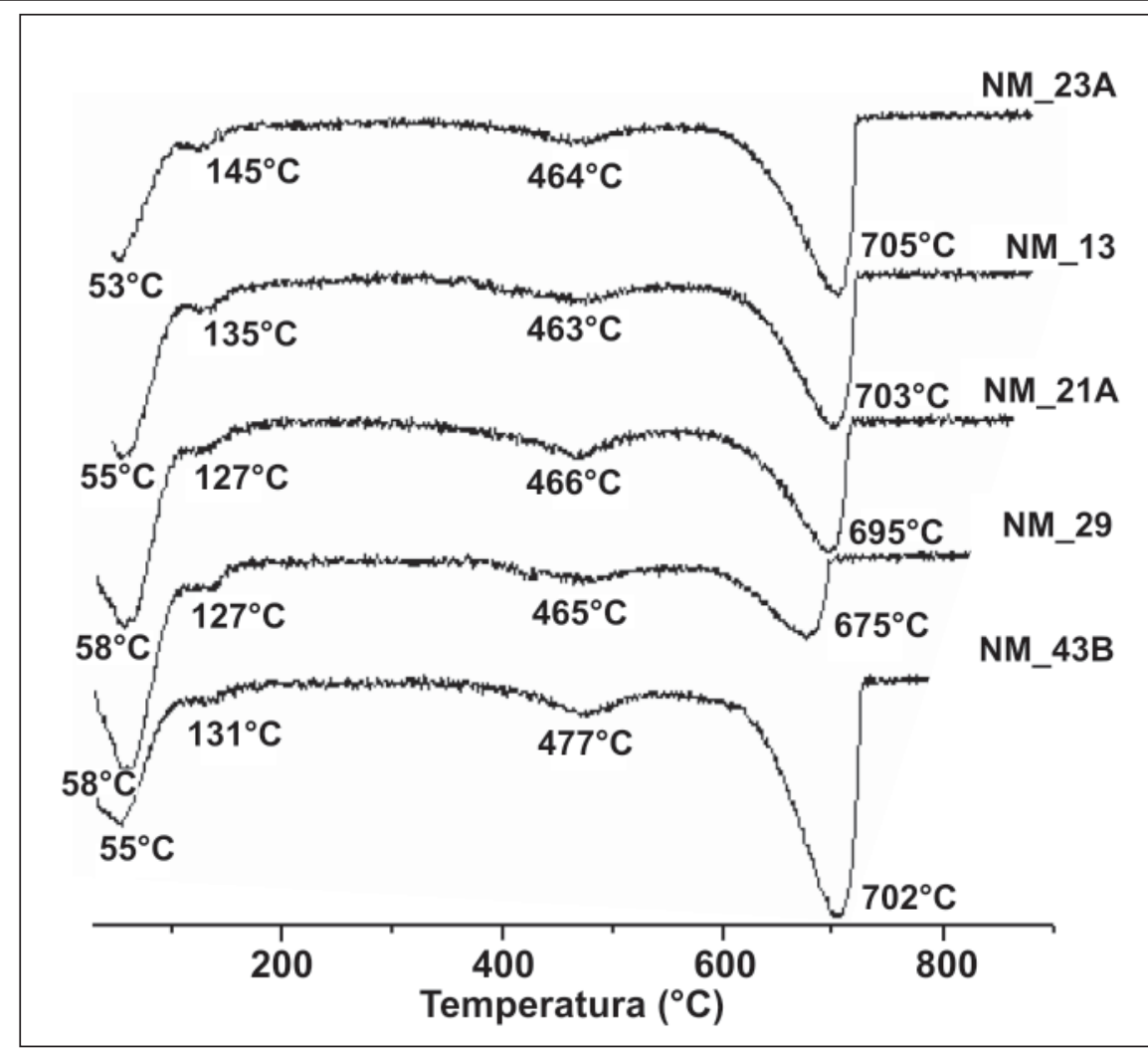

Figura 5 - Curvas termodiferenciais (ATD) das amostras de lutitos diatomáceos, mostrando a presença de picos endotérmicos durante o aquecimento das amostras até $900^{\circ} \mathrm{C}$.

Tabela 2 - Valores de densidade, superfície específica e volume total de poros das cinco amostras naturais de lutitos diatomáceos.

\begin{tabular}{c|c|c|c|c}
\hline Amostras & $\begin{array}{c}\text { Densidade } \\
\text { Aparente } \\
\left(\mathbf{g} / \mathbf{c m}^{3}\right)\end{array}$ & $\begin{array}{c}\text { Densidade } \\
\left(\mathbf{g} / \mathbf{c m}^{3}\right)\end{array}$ & $\begin{array}{c}\text { Superfície } \\
\text { Específica - } \\
\text { BET }\left(\mathbf{m}^{2} / \mathbf{g}\right)\end{array}$ & $\begin{array}{c}\text { Volume Total } \\
\text { de Poros } \\
\left(\mathbf{c m}^{3} / \mathbf{g}\right)\end{array}$ \\
\hline NM_23A & 1,84 & 2,25 & 20,24 & 0,041 \\
\hline NM_13 & 1,94 & 2,07 & 17,18 & 0,076 \\
\hline NM_21A & 1,85 & 2,28 & 8,44 & 0,033 \\
\hline NM_29 & 2,06 & 2,37 & 20,04 & 0,001 \\
\hline NM_43B & 1,90 & 2,25 & 8,11 & 0,022 \\
\hline
\end{tabular}

tura média de $700^{\circ} \mathrm{C}$. As relações molares calculadas entre as porcentagens dos óxidos totais da mistura (Tabela 1) foram as seguintes: $\mathrm{SiO} / \mathrm{Al}_{2} \mathrm{O}_{3}=8,24$ e $\mathrm{Na}_{2} \mathrm{O} / \mathrm{Al}_{2} \mathrm{O}_{3}=0,26$. A relação molar $\mathrm{Na}_{2} \mathrm{O} / \mathrm{Al}_{2} \mathrm{O}_{3}$ foi modificando-se até 1,1 , motivada pela adesão da solução alcalina de $\mathrm{NaOH}$ a $15 \mathrm{~mol} / \mathrm{l}$ durante a fabricação do geopolímero (Figura 7). Comparando com as faixas de valores empregadas por Davidovits (1982), nessa pesquisa foram utilizadas relações molares $\mathrm{SiO} / \mathrm{Al}_{2} \mathrm{O}_{3}$ maiores do que 4 e $\mathrm{Na}_{2} \mathrm{O} / \mathrm{Al}_{2} \mathrm{O}_{3}$ na faixa de 1,0 a 1,14 . As resistências logradas em ensaios simples de compressão foram até $7 \mathrm{Mpa}$. 
Miguel Genaro Peralta Sánchez et al.

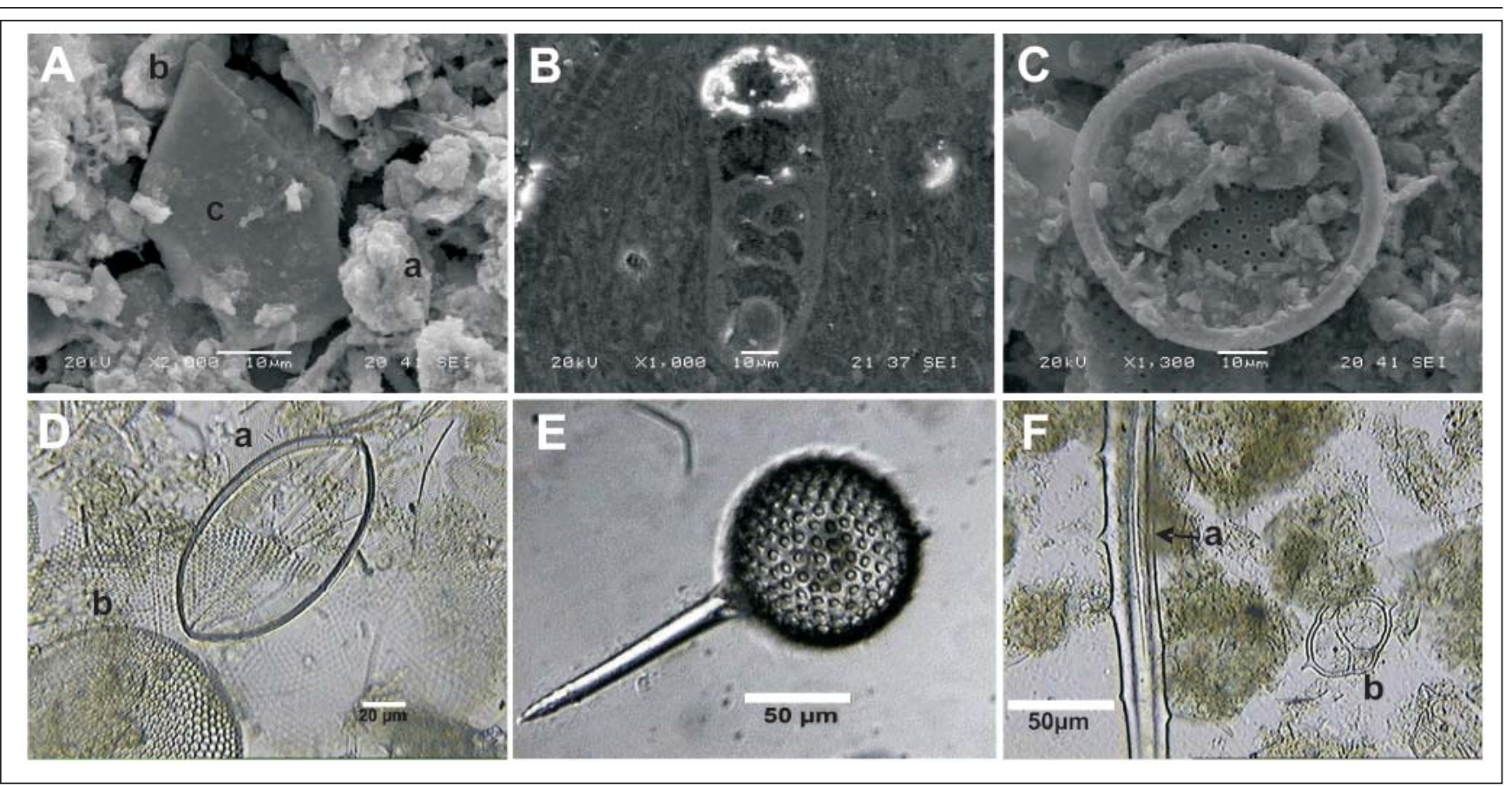

Figura 6 - Imagens obtidas no MEV (A,B,C) e Microscópio Ótico (D,E,F) dos lutitos diatomáceos, sendo, A: Fragmentos: (a) montmorilonita formando aglomerados (b) calcita e (c) quartzo autigênico; B: Foraminífero em matriz silicoaluminosa; C: Diatomácea do tipo centrales; D: (a) diatomácea tipo pennales (b) diatomácea tipo centrales; E: Radiolario tipo Spumellaria sp; F: (a) Espícula e (b) Silicoflagelado tipo Distephanus sp.
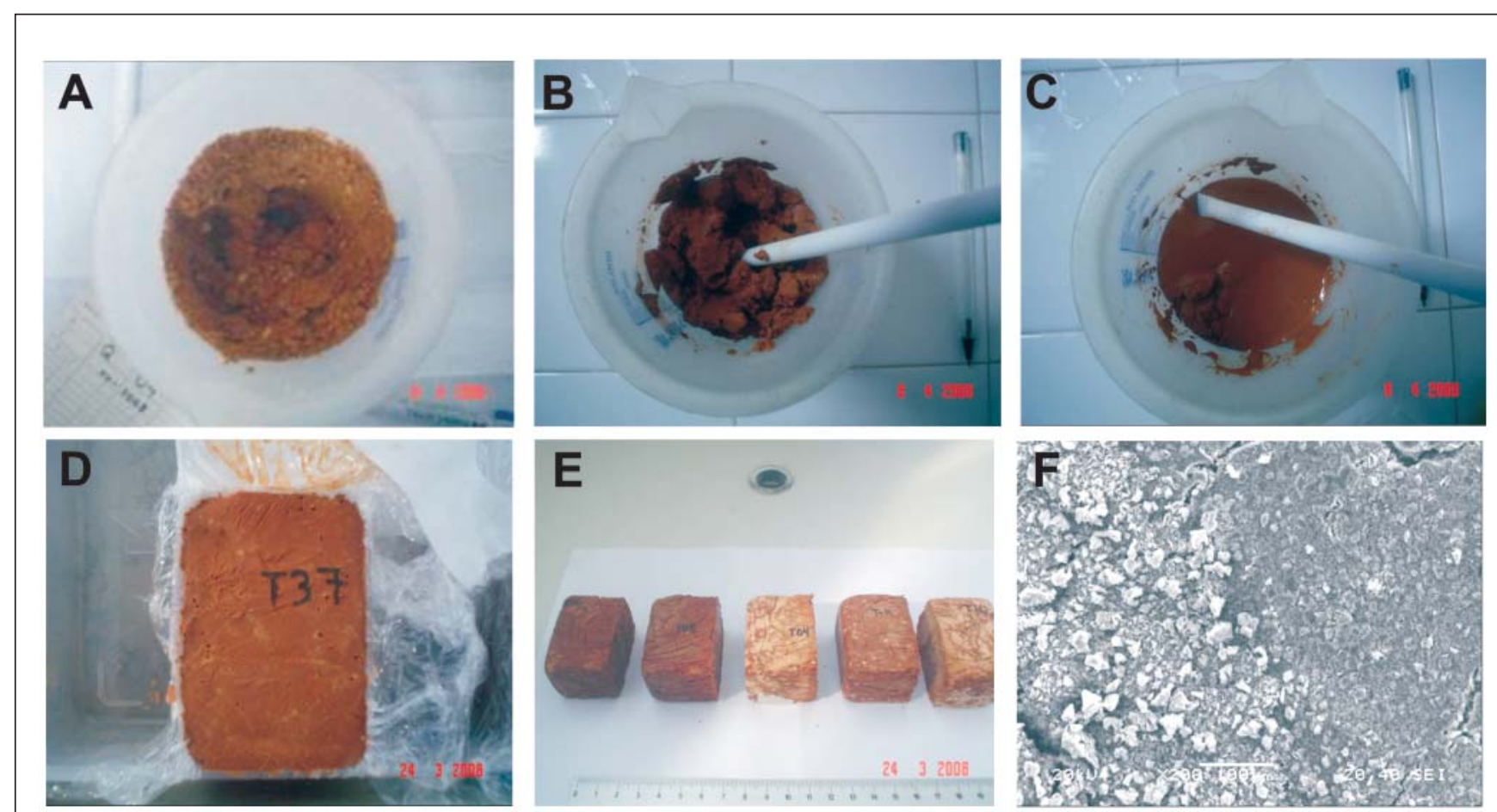

Figura 7 - Fotografias do processo de fabricação de geopolímeros. A: lutito diatomáceo moído e aquecido, em recipiente plástico para mistura. B) amostra umedecida com solução de $\mathrm{NaOH}$ e homogeneizada com ajuda de bastão de teflon. C) amostra reagindo e formando o gel. D) geopolímero colocado no molde plástico, vedado e após três horas em estufa a $45^{\circ} \mathrm{C}$. E: blocos de geopolímeros de oito dias. F: superfície geopolimérica com alguns resíduos sobre a matriz. 


\section{Conclusões}

A pesquisa dos lutitos diatomáceos permitiu conhecer as suas principais características químicas e físicas e alguns aspectos relevantes como a baixa densidade aparente e seu baixo conteúdo de carapaças de diatomáceas.

Os menores valores de umidade natural, em média de $5,4 \%$, foram encontrados na área A03, localizada em San Antonio, enquanto os valores mais elevados, em média $8 \%$, foram registrados na área 01, localizada em Sucre e na área 02 ao sul de Olmedo.

A distribuição granulométrica das amostras indicou altos valores da fração silte, situados entre 57 e 90\%. Observações ao MEV demonstraram que parte do teor de silte foi gerada pela cimentação carbonática de aglomerados de argila montmorilonítica.

A mineralogia das amostras coletadas indicou a presença de calcita, quartzo, feldspatos, montmorilonita, caulinita e micas, além de zeólitas encontradas como traços. Picos endotérmicos de montmorilonita e de carbonatos foram identificados por análises termogravimétricas.

A composição química das amostras indicou predominância da sílica, relacionada com a presença de quartzo, feldspatos e da sílica amorfa oriunda de fósseis silicosos. Os altos percentuais de alumina foram relacionados à presença do argilominerais montmoriloníticos, enquanto os teores de $\mathrm{CaO}$ são devidos à presença de carbonatos e de foraminíferos.

Os valores de densidade obtidos nas amostras são próximos dos da sílica amorfa. A superfície específica, obtida pelo método de BET, mostrou valores baixos, que são influenciados, principalmente, pela presença de carbonatos, quartzo e feldspato.

O estudo morfológico dos constituintes da biota, realizado com microscopia eletrônica de varredura, permitiu identificar, principalmente, foraminíferos e diatomáceas. Ao microscópio ótico, observou-se que os microfósseis silicosos ocorrem imersos numa matriz silico-aluminosa-carbonática, o que dificulta sua concentração para análises mais detalhadas. O preenchimento das cavidades das diatomáceas pela matriz restringe a possibilidade de sua utilização industrial na fabricação de filtros. Entretanto, devido ao seu conteúdo sílico-aluminoso, os lutitos diatomáceos pesquisados podem ser utilizados como fonte de sílica e alumina para diversas aplicações como solo-cimento, geopolímeros, refratários e zeólitas sintéticas.

A geopolimerização da mistura de diferentes amostras de lutitos diatomáceos permite viabilizar sua utilização na fabricação de aglomerante mediante a ativação alcalina. Os resultados de resistência mecânica dos geopolímeros, apesar de considerados baixos, poderiam ser modificados mediante outras pesquisas que possibilitem a mudança de variáveis, como adições de outras fontes de alumina, tipo e concentração dos reagentes, tamanho de grão, temperatura e tempo de aquecimento, para melhorar as características mecânicas dos produtos geopoliméricos.

\section{Agradecimentos}

Os autores expressam seus agradecimentos ao Conselho Nacional de Desenvolvimento Científico e Tecnológico $(\mathrm{CNPq})$ pela concessão de bolsa de estudo ao primeiro autor, à Universidade Federal de Ouro Preto-UFOP pela utilização dos laboratórios e à empresa Geologia e Sondagem - Geossol pela realização de análises laboratoriais. Da mesma forma, os autores externam o seu reconhecimento à Profa. Dra. Rubia Ribeiro Viana - UFMT pelà realização de análises químicas. No Equador, à Escuela Superior Politécnica del Litoral, ao Componente 6 do Programa VLIR/ ESPOL e ao Centro de Investigación Científica y Tecnológica (CICYT) pela colaboração nos trabalhos de campo e aos laboratórios das Faculdades de Ingeniería en Ciencias de la Tierra (FICT) e Ingeniería en Mecánica y Ciencias de la Producción (FIMCP).

\section{Referências} bibliográficas

ANGLO-ECUADORIAN OILFIELDS. Guidebook of the Geology of the Santa Elena Peninsula. Santa Elena-Ecuador Ecuadorian Geological and Geophysical Society, 1970. 36p. (Relatório técnico).

ARRATA, L., BOSSE, H., MYLIUS, H. Investigaciones de materias primas minerales no metálicas en el Ecuador. Quito: INEMIN \& BGR. v. 5, 1990. 242p. (Relatório técnico).

BALDOCK, J. W. Geologia del Ecuador. Boletín de la Explicación del Mapa Geológico de la República del Ecuador. Escala 1:1'000.000. Quito - DDGGM (hoje CODIGEM), Londres: IGS, 1982. 66p. (Boletim).

BENITEZ, S. Evolution géodynamique de la province cotière sud-équatorienne au Cretacé supèrieur Tertiare. Labo. Chaines Alpines, Grenoble, 1995, 221p. (Thèse).

BRITO, S., MARKWICH, H. Bloques sílico-calcáreos: una alternativa para la industria de la construcción. Minería ecuatoriana, Quito, 2. p. 27-30, 1990.

BRUNAUER, S., EMMETT, P. H., TELLER, E. Adsorption of Gases in Multimolecular Layers. Journal of American Chemical Society, n. 60, p.309-319, 1938.

CARBALLO, F. E. Las zeolitas de la costa de Ecuador (Guayaquil): geología, caracterización y aplicaciones. Madrid: ETSI-MINAS, Universidad Politécnica de Madrid, 1994. 386p. (Tese de Doctorado).

CUSHMAN, J. A. Foraminifera: their classification and economic use. London: Harvard University Press, 1976. $605 \mathrm{p}$.

DAVIDOVITS, J. Mineral polymers and methods of making them. U.S. patent 4.349.386, 1982.

DENIAUD, Y. Evolucion TectonoSedimentaria de las cuencas costeras Neógenas del Ecuador. Fonds (IRD) Documentaire ORSTOM, Quito, 1998, 69p.

DENIAUD, Y., BABY, P., BASILE, C., ORDOÑEZ, M., MASClE, G., MONTENEGRO, G. Neogene evolution of the main ecuadorian fore-arc sedimentary basins and sediment mass-balance inferences. Fourth / SAG, Goettingen (Germany), p. 201215, 1999.

DORFMAN, M. H. A plate tectonic model for development of the geology and estimation of potential petroleum reserves in equador. Zagreb, 1976. 35p. (Relatório Técnico). 
EARTH SCIENCE DATA INTERFACE (ESDI). Imagen Tif Landsat. In http:// glcfapp.umiacs.umd.edu:8080, 2004.

EILERT, V. P., BAECKER-FAUTH, S., SOUZA V. Radiolários. In: Paleontologia Carvalho I. S. São Paulo: Editora Interciencia, 2004. p. 297-312. 861p.

EMBRAPA - Pesquisa Brasileira de Pesquisa Agropecuária. Manual de Métodos de Análises de Solo, (2. ed.) Rio de Janeiro: EMBRAPA, 1997. 212p.

FEININGER, T., BRISTOW, C. R. Cretaceous and Paleocene geologic history of coastal Ecuador. Geologische Rundschau, 69, p.849-874, 1980.

GOOSENS P. J., ROSE, W. I. Chemical composition and age determination of tholeitic rocks in the Basin Cretaceous Complex. Ecuador: GSA Bulletin, v. 84, p. 1043-1052, 1973.

GREENE-KELLY, R. The identification of montmorillonoids in clays. Journal of Soil Science, n. 4, p. 233-237, 1953.

HIGLEY, D. K. The Progreso Basin Province of Northwestern Peru and Southwestern Ecuador: neogene and cretaceous-paleogene total petroleum systems. in http://pubs.usgs.gov/bul/ b2206-b/, U.S.G.S., Virginia. 2004. 30p.

HOLDERBANK. Geological and chemical investigations and results of the overall raw prospection for the CEMEC. Cement Plant Project, Guaiaquil, 1974. 173p. (Relatório Técnico).

INSTITUTO GEOGRÁFICO MILITAR (IGM). Mapa geológico del Ecuador. Folha 17 - Estero Salado, escala 1:100.000, 1968.

INSTITUTO GEOGRÁFICO MILITAR (IGM). Mapa topográfico da Península de Santa Elena. Folha MV - AS 17 - 10, escala 1:250.000, 1984.
JAILLARD, È., ORDOÑEZ, M. BERRONES, S., JIMÉNEZ, N., MONTENEGRO, G., ZAMBRANO, I. Basin development in na accretionary, oceanic-floored fore-arc setting: southern coastal Ecuador during Late Cretaceios-Late Eocene time. AAPG Memoir 62, p. 615-631, 1995.

JUTEAU, T., MÉGARD, F., RAHARISON, L., WHITECCHURCH, H. Les assemblage ophiolitique de l'occident equatorien: nature petrographique et position structurale. Boll, Soc, Geol, France, t, XIX, n. 5, p. 1125-1132, 1977.

LONSDALE, P. Ecuadorian Subduction System. AAPG Bulletin, v. 62, n. 12, p. 24542477, 1978.

MACKENZIE, R. C. The differential thermal investigation of clays. London: The Central Press, 1957. 456p.

MÉGARD, F., ROPERCH, P., LEBRAT, M., LAJ, C., MOURIER, T., NOBLET, C. L'occident equatorien: un terrain océanique pacifique accolé au continent sud-américain. Bull. Inst. Fr. And. XVI, n. 1-2, p. 39-54, 1987.

MORALES-CARRERA, A. M. Caracterização mineralógica das argilas da Península de Santa Elena, Equador. Ouro Preto: Escola de Minas, Universidade Federal de Ouro Preto, 2003. 89p. (Dissertação de Mestrado).

MORALES-CARRERA, A. M., VARAJÃO, A. F. D. C., GONÇALVES, M. A. Caracterização mineralógica das argilas da Península de Santa Elena, Equador. REM-Revista da Escola de Minas, v. 1, n. 61, p.95-103, 2008.

ORDOÑEZ, M., JIMÉNEZ, N., SUÁREZ, J. Micropaleontología ecuatoriana, datos bioestratigráficos y paleontológicos de las cuencas: Graben de Jambelí, Progreso, Manabí, Esmeraldas y Oriente; del levantamiento de la Península de Santa Elena, y de las cordilleras Chongón Colonche, costera y occidental. Petroproducción. Guayaquil: CIGG, 2006. 634p.

ORDOÑEZ, M., ZAMBRANO, I., JIMÉNEZ, N. Bioestratigrafía de la Cuenca Progreso en la provincia del Guayas. In: CONGRESSO ECUATORIANO DE G. M. P., 4. Quito, 1986. 50p.

PERALTA-SÁNCHEZ, M. G. Caracterização mineralógica e tecnológica dos lutitos diatomáceos da Península de Santa Elena, Equador. Ouro Preto: Escola de Minas, Universidade Federal de Ouro Preto, 2008. 78p. (Dissertação de Mestrado).

RAMOS, V. Caracterización y preparación de dos muestras de diatomita para su utilización como filtros, pinturas y bloques alivianados. Guayaquil: Facultad de Ingeniería en Ciencias de la Tierra, Escuela Superior Politécnica del Litoral, 1996. 101p. (Tese de Graduação).

VILELA, C. G. Diatomáceas. In: Paleontologia, Carvalho I. S. São Paulo: Editora Interciencia, 2004. p. 319-326. 861 p.

WALKER, G. F. Reactions of expanding lattice clay minerals with glycerol and ethyelne glycol. Clay Mineral. Bulletin, n. 3, p. 302, 1958.

XU, H., VAN DEVENTER, J.S.J. The geopolymerisation of alumino-silicate minerals. Int. J. Miner. Process. v. 59, p. 247-266, 2000.

Artigo recebido em 26/03/2009 e aprovado em 09/12/2009.

\section{A REM tem novo endereço: FUNDAÇÃO GORCEIX - REM Rua Carlos Walter Marinho Campos, 57 Bairro: Vila Itacolomy 35400-000 - Ouro Preto - MG

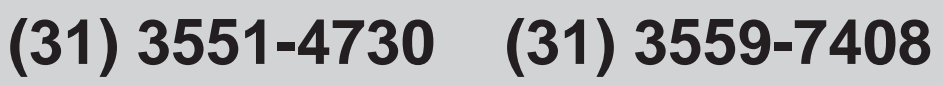 www.rem.com.br}

